TRANSACTIONS OF THE

AMERICAN MATHEMATICAL SOCIETY

Volume 364, Number 6, June 2012, Pages 3137-3158

S 0002-9947(2012)05459-6

Article electronically published on January 31,2012

\title{
BUNDLES OF COLOURED POSETS AND A LERAY-SERRE SPECTRAL SEQUENCE FOR KHOVANOV HOMOLOGY
}

\author{
BRENT EVERITT AND PAUL TURNER
}

\begin{abstract}
The decorated hypercube found in the construction of Khovanov homology for links is an example of a Boolean lattice equipped with a presheaf of modules. One can place this in a wider setting as an example of a coloured poset, that is to say, a poset with a unique maximal element equipped with a presheaf of modules. In this paper we initiate the study of a bundle theory for coloured posets, producing for a certain class of base posets a Leray-Serre type spectral sequence. We then show how this theory finds an application in Khovanov homology by producing a new spectral sequence converging to the Khovanov homology of a given link.
\end{abstract}

\section{INTRODUCTION}

Our motivation for studying coloured posets comes from a desire to better understand some of the structures arising in the Khovanov homology of links [1, 4. One can ask if Khovanov's "cube" construction can be placed in a broader context, hoping that this new perspective allows one to lever some advantage. In 2 we showed that this is possible using the framework of what we call coloured posets. This is something of a half-way house between the specifics of "cubes" and the full generality of presheaves of modules over small categories. We believe this is an appropriate place to study Khovanov homology as it provides enough generality to be useful, without losing sight of the starting point, the idea being that one can then bring the algebraic topology of coloured posets into the game.

One direction of interest is a bundle theory for coloured posets which we initiate in this paper. Given a poset $B$ with a unique maximal element, we define bundles of coloured posets over $B$. Roughly, we wish to capture the idea that a coloured poset may be decomposed as a family of coloured posets parametrized by another poset, much as a fibre bundle is a family of spaces parametrized by some base space. A bundle of coloured posets has a total coloured poset $(E, \mathcal{F})$, and our central interest

Received by the editors January 16, 2009 and, in revised form, August 25, 2010.

2010 Mathematics Subject Classification. Primary 57M27; Secondary 06A11, 55T10.

Key words and phrases. Coloured poset, spectral sequence, poset bundle, Khovanov homology.

The first author thanks Finnur Larusson for many useful and stimulating discussions. He is also grateful to the Alpine Mathematical Institute, Haute-Savoie, France, and to the Institute for Geometry and its Applications, University of Adelaide, Australia.

The second author was partially supported by the Swiss National Science Foundation projects no. 200020-113199 and no. 200020-121506.

(C)2012 American Mathematical Society Reverts to public domain 28 years from publication 
is in computing its homology. Our main result in this direction, proved as Theorem 5.1 in 95 , is a Leray-Serre style spectral sequence for bundles where the base has a certain technical property that we call special admissibility:

Main Theorem. Let $\xi: B \rightarrow \mathcal{C P}_{R}$ be a bundle of coloured posets with $B$ finite and specially admissible, and let $(E, \mathcal{F})$ be the associated total coloured poset. Then there is a spectral sequence

$$
E_{p, q}^{2}=H_{p}\left(B, \mathcal{H}_{q}^{\mathrm{fib}}(\xi)\right) \Longrightarrow H_{*}(E, \mathcal{F}) .
$$

It turns out that a number of naturally occurring posets are specially admissible, and importantly for applications to knot homology theories, this class includes the Boolean lattices. Given a knot diagram $D$ together with $k$ distinguished crossings $c_{1}, \ldots, c_{k}$, one can obtain a new complex in the spirit of Khovanov: resolving each of the remaining $\ell$ crossings gives another link diagram, and the $\ell$ diagrams that result can be placed as the vertices of a hypercube (a Boolean lattice). Taking the unnormalised Khovanov homology of these diagrams and using induced maps along edges one obtains, by the construction of Khovanov, a triply graded complex whose homology we denote by $\overline{K H}_{*, *, *}\left(D ; c_{1}, \ldots, c_{k}\right)$.

Main Application. Let $D$ be a link diagram and let $c_{1}, \ldots, c_{k}$ be a subset of the crossings of $D$. For each $i$, there is a spectral sequence

$$
E_{p, q}^{2}=\overline{K H}_{p, q, i}\left(D ; c_{1}, \ldots, c_{k}\right) \Longrightarrow \overline{K H}_{p+q, i}(D) .
$$

The $r$-th differential in the spectral sequence has bi-degree $(-r, r-1)$.

The paper unfolds as follows. In the next section we define bundles of coloured posets, describe the associated total coloured poset, and give a number of examples. We also recollect facts from [2] about coloured posets and their homology. In \$2 we start with a bundle of coloured posets and introduce a certain bicomplex which (by standard methods) gives rise to a spectral sequence converging to the homology of the total complex. Our task later will be to recognise this as the homology of the total coloured poset of the bundle we started with. In fact we can only do this for a restricted class of bases, the specially admissible ones, and we introduce these in 93 . We give a number of examples, including the Boolean lattices. Following this, 84 introduces the technical tools needed to prove the main theorem, namely certain long exact sequences. In $\$ 5$ we state and prove the main theorem. Finally in $\$ 6$ we turn to Khovanov homology and construct a new spectral sequence which for a given link has a potentially computable $E^{2}$-page, and which converges to the Khovanov homology of the link.

\section{Bundles of COLOURED POSETS}

Recall from [2] that a coloured poset is a pair $(P, \mathcal{F})$ consisting of a poset $P$ having a unique maximal element $1_{P}$, and a covariant functor $\mathcal{F}: P \rightarrow \mathcal{M}_{R}$, called the colouring. This may be regarded as a representation of $P$ or as a presheaf of modules over $P$ according to taste. A morphism of coloured posets $\left(P_{1}, \mathcal{F}_{1}\right) \rightarrow\left(P_{2}, \mathcal{F}_{2}\right)$ is a pair $(f, \tau)$, where $f: P_{1} \rightarrow P_{2}$ is a map of posets, and $\tau=\left\{\tau_{x}\right\}_{x \in P_{1}}$ is a collection of $R$-module homomorphisms $\tau_{x}: \mathcal{F}_{1}(x) \rightarrow \mathcal{F}_{2}(f(x))$. This pair must satisfy (i) $f(x)=1_{P_{2}}$ if and only if $x=1_{P_{1}}$, and (ii) for all $x \leq y$ in $P_{1}$, the following diagram 
commutes:

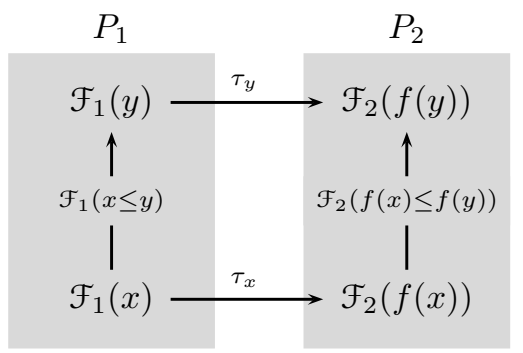

Coloured posets and morphisms between them form the category $\mathcal{C P}_{R}$.

We also recall from [2] our poset convention that if $P$ has a unique minimal element $0_{P}$, then $0_{P}<1_{P}$. In particular the poset with a single element has a 1 but not a 0 . If $x<y$ in $P$ and there is no $z$ with $x<z<y$, then one says that $y$ covers $x$ and writes $x \prec y$.

Throughout this paper, if $x \in P$ we write $P(x)$ for the interval

$$
P(x):=\{y \in P \mid y \leq x\}
$$

and $\bar{P}(x)$ for its complement

$$
\bar{P}(x):=\{y \in P \mid y \not \leq x\} .
$$

From now on we will just say "poset with 1 " to refer to a poset with a unique maximal element.

The idea of a bundle is that it consists of a collection of coloured posets (the fibres) parametrized by another poset (the base) that encodes instructions for gluing the fibres together.

Definition 1.1. Let $B$ be a poset with unique maximal element $1_{B}$. A bundle of coloured posets with base $B$ is a (covariant) functor $\xi: B \rightarrow \mathcal{C P}_{R}$.

Thus, to each $x \in B$ there is an associated coloured poset $\xi(x)=\left(E_{x}, \mathcal{F}_{x}\right)$, the fibre over $x$. Whenever $x \leq z$ there is an associated morphism of coloured posets $\xi(x \leq z)=\left(f_{x}^{z}, \tau_{x}^{z}\right)$ from $\xi(x)$ to $\xi(z)$. There is a projection map of (uncoloured) posets $\pi: E \rightarrow B$ defined by $\pi(y)=x$ if and only if $y \in P_{x}$. Definition 1.1 is akin to the interpretation of a vector bundle (with connection) as a functor from the path space of the base to the category of vector bundles. What is missing from this definition is the analogue of the total space, which is somewhat hidden but which can be constructed as follows.

Definition 1.2. Let $B$ be a poset with 1 and let $\xi: B \rightarrow \mathcal{C P}_{R}$ be a bundle of coloured posets. The associated total coloured poset is the coloured poset $(E, \mathcal{F})$ defined by:

- As a set, $E$ is equal to $\bigcup_{x \in B} E_{x}$, the union of the fibres, with the partial order on $E$ defined as follows:

- if $y, y^{\prime} \in E_{x}$, so $y$ and $y^{\prime}$ are in the same fibre, then $y \leq y^{\prime}$ iff $y \leq y^{\prime}$ in $E_{x}$,

- if $y \in E_{x}$ and $y^{\prime} \in E_{x^{\prime}}$ with $x \neq x^{\prime}$, then $y \leq y^{\prime}$ iff $x \leq x^{\prime}$ in $B$ and $f_{x}^{x^{\prime}}(y) \leq y^{\prime}$ in $E_{x^{\prime}}$.

- The colouring $\mathcal{F}: E \rightarrow \mathcal{C P}_{R}$ is defined on an object $y$ in fibre $E_{x}$ by setting $\mathcal{F}(y)=\mathcal{F}_{x}(y)$, and on a morphism $y \leq y^{\prime}$ as follows:

- if $y, y^{\prime} \in E_{x}$, then $\mathcal{F}\left(y \leq y^{\prime}\right)=\mathcal{F}_{x}\left(y \leq y^{\prime}\right)$, 
- if $y \in E_{x}$ and $y^{\prime} \in E_{x^{\prime}}$ with $x \neq x^{\prime}$, then

$$
\mathcal{F}\left(y \leq y^{\prime}\right)=\mathcal{F}_{x^{\prime}}\left(f_{x}^{x^{\prime}}(y) \leq y^{\prime}\right) \circ\left(\tau_{x}^{x^{\prime}}\right)_{y} .
$$

In the last line of the definition we are using the morphism of coloured posets $\xi\left(x \leq x^{\prime}\right)=\left(f_{x}^{x^{\prime}}, \tau_{x}^{x^{\prime}}\right)$ and recalling that $\left(\tau_{x}^{x^{\prime}}\right)_{y}$ is a map $\mathcal{F}_{x}(y) \rightarrow \mathcal{F}_{x^{\prime}}\left(f_{x}^{x^{\prime}}(y)\right)$.

Lemma 1.3. The pair $(E, \mathcal{F})$ of Definition 1.2 is a coloured poset.

Proof. It is easily checked that $E$ is a poset. For the colouring we must verify that composition behaves as it should. There are four cases to consider of which we treat one in detail, the others being much simpler.

Let $y \in E_{x}, y^{\prime} \in E_{x^{\prime}}$ and $y^{\prime \prime} \in E_{x^{\prime \prime}}$, where $x \neq x^{\prime} \neq x^{\prime \prime}$. Suppose that $y \leq y^{\prime}$ and $y^{\prime} \leq y^{\prime \prime}$. We must show that $\mathcal{F}\left(y^{\prime} \leq y^{\prime \prime}\right) \circ \mathcal{F}\left(y \leq y^{\prime}\right)=\mathcal{F}\left(y \leq y^{\prime \prime}\right)$. Consider the following diagram:

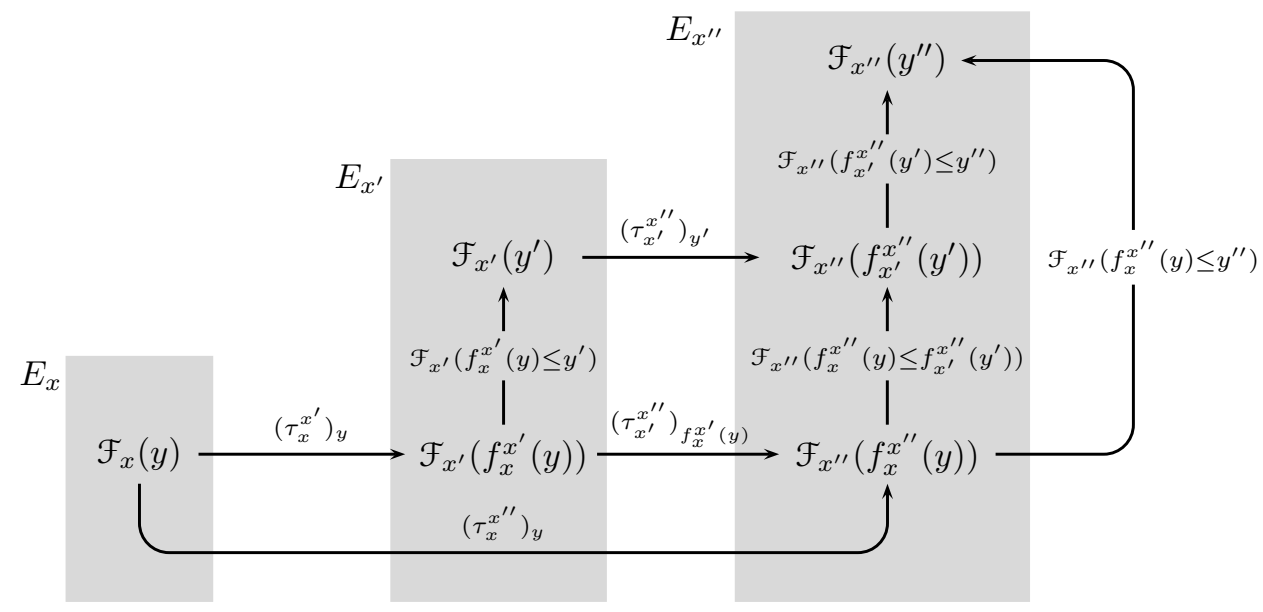

The triangle at the bottom commutes since $\xi$ is a functor, the triangle at the right commutes because $\mathcal{F}_{x^{\prime \prime}}$ is a functor and the square commutes by the naturality of $\tau_{x^{\prime}}^{x^{\prime \prime}}$. Thus the entire diagram commutes. Going up the steps gives

$$
\mathcal{F}\left(y^{\prime} \leq y^{\prime \prime}\right) \circ \mathcal{F}\left(y \leq y^{\prime}\right)
$$

while following the two arrows with bends gives $\mathcal{F}\left(y \leq y^{\prime \prime}\right)$.

Example 1.4. Figure 1 gives an example of a bundle over a Boolean lattice of rank two, with the colouring left off.

Example 1.5. Let $B$ be a poset with 1 and let $(P, \mathcal{F})$ be a coloured poset. The product bundle with base $B$ and fibre $(P, \mathcal{F})$, denoted $B \times(P, \mathcal{F})$, is defined by the functor $\xi: B \rightarrow \mathcal{C P}_{R}$, where $\xi(x)=(P, \mathcal{F})$ and $\xi\left(x_{1} \leq x_{2}\right)=$ id.

Example 1.6. Let $B$ be the Boolean lattice of rank 1, i.e., the poset with two elements 0,1 and $0<1$, and let $\xi$ be a bundle over $B$. The associated total coloured poset $(E, \mathcal{F})$ is the result of gluing the coloured posets $\left(P_{0}, \mathcal{F}_{0}\right)$ and $\left(P_{1}, \mathcal{F}_{1}\right)$ along the morphism

$$
\left(f_{0}^{1}, \tau_{0}^{1}\right):\left(P_{0}, \mathcal{F}_{0}\right) \rightarrow\left(P_{1}, \mathcal{F}_{1}\right),
$$

as defined in [2, §1]. 


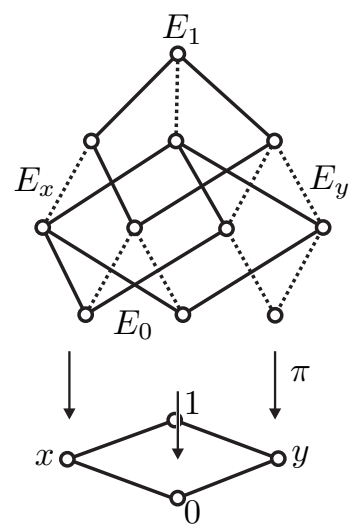

Figure 1. Example of a bundle with the colouring omitted.

Example 1.7. Let $X$ be a set, $\mathbb{B}(X)$ the Boolean lattice of all subsets of $X$, and $\mathcal{F}: \mathbb{B}(X) \rightarrow \mathcal{M}_{R}$ a colouring. If $A \in \mathbb{B}(X)$, then $(\mathbb{B}(X), \mathcal{F})$ decomposes as a bundle with base $\mathbb{B}(X \backslash A)$ and each fibre a copy of $\mathbb{B}(A)$ : a $B \in \mathbb{B}(X)$ lies in the fibre over $B \cap(X \backslash A)$ at the element $B \cap A \in \mathbb{B}(A)$, and is coloured $\mathcal{F}(B)$. This is just a decomposition of $\mathbb{B}(X)$ as a product of posets $\mathbb{B}(X \backslash A) \times \mathbb{B}(A)$. If $Y \in \mathbb{B}(X \backslash A)$, then $E_{Y}=\{Y \cup Z \mid Z \in \mathbb{B}(A)\}$ and $Y \cup Z \mapsto Y^{\prime} \cup Z$ is the poset map $f_{Y}^{Y^{\prime}}: E_{Y} \rightarrow E_{Y^{\prime}}$ when $Y \leq Y^{\prime} \in \mathbb{B}(X \backslash A)$, with $\left(\tau_{Y}^{Y^{\prime}}\right)_{Y \cup Z}$ equal to the map $\mathcal{F}\left(Y \cup Z \leq Y^{\prime} \cup Z\right)$.

Example 1.8. If $B$ is a poset with 1 , and $\xi$ is a bundle over $B$, then any sub-poset $A \subseteq B$ having a unique maximal element has an associated bundle obtained by restricting $\xi$. This is given by the composition of functors $A \rightarrow B \rightarrow \mathcal{C P}_{R}$ and will be denoted by $\left.\xi\right|_{A}$. Note that the maximal element of $A$ need not be the maximal element in $B$. Two instances of particular importance arise when $\xi$ is a bundle over $B$ with total coloured poset $(E, \mathcal{F})$, and for $x \in B$ we consider the subposets to be the interval $B(x)$ and its complement $\bar{B}(x)$. The interval $B(x)$ has maximal element $x$ and the complement has maximal element the 1 of $B$. We will denote the total coloured posets of the restricted bundles $\left.\xi\right|_{B(x)}$ and $\left.\xi\right|_{\bar{B}(x)}$ by $(E(x), \mathcal{F})$ and $(\bar{E}(x), \mathcal{F})$.

Bundles of coloured posets form a category $\mathcal{B} u d_{R}$ as we now describe.

Definition 1.9. Let $\xi: B \rightarrow \mathcal{C P}_{R}$ and $\xi^{\prime}: B^{\prime} \rightarrow \mathcal{C P}_{R}$ be bundles of coloured posets over $B$ and $B^{\prime}$ respectively. A morphism $(g, \eta): \xi \rightarrow \xi^{\prime}$ is a map of (uncoloured) posets $g: B \rightarrow B^{\prime}$ satisfying $g(x)=1$ iff $x=1$, together with a natural transformation $\eta$ from $\xi$ to $\xi^{\prime} \circ g$.

By fixing the base poset $B$ and restricting morphisms to those with $g=\mathrm{id}$, one obtains a subcategory of bundles over $B$, denoted $\mathcal{B} u n d_{R}(B)$.

Associating the total coloured poset to a bundle is functorial: given a bundle $\xi: B \rightarrow \mathcal{C P}_{R}$ let $\mathcal{E}(\xi)=(E, \mathcal{F})$ be the associated total coloured poset. For a morphism $(g, \eta): \xi \rightarrow \xi^{\prime}$ between bundles $\xi: B \rightarrow \mathcal{C P}_{R}$ and $\xi^{\prime}: B^{\prime} \rightarrow \mathcal{C P}_{R}$, we define a morphism of coloured posets $\mathcal{E}(g, \eta): \mathcal{E}(\xi) \rightarrow \mathcal{E}\left(\xi^{\prime}\right)$ in the following way. Recall that we must define a poset map $f: E \rightarrow E^{\prime}$ and a collection $\left\{\sigma_{y}: \mathcal{F}(y) \rightarrow\right.$ $\left.\mathcal{F}^{\prime}(f(y))\right\}$, and that for $x \in B$, the natural transformation $\eta$ gives a morphism of 
coloured posets,

$$
\eta(x):=\left(f_{x}^{g(x)}, \tau_{x}^{g(x)}\right): \xi(x) \rightarrow \xi^{\prime} g(x),
$$

where $\tau_{x}^{g(x)}$ is the collection of morphisms $\left\{\left(\tau_{x}^{g(x)}\right)_{y}\right\}_{y \in \xi(x)}$. Define $f: E \rightarrow E^{\prime}$ by $f(y)=f_{x}^{g(x)}(y)$ for $y \in \xi(x)$, and $\sigma_{y}=\left(\tau_{x}^{g(x)}\right)_{y}$.

If $x \leq z \in B$ we have the commuting diagram

$$
\begin{array}{cc}
\xi(z) \stackrel{\eta(z)}{\longrightarrow} \xi^{\prime} g(z) & \uparrow \\
\left(f_{x}^{z}, \tau_{x}^{z}\right) \uparrow & \uparrow_{\left(f_{g(x)}^{g(z)}, \tau_{g(x)}^{g(z)}\right)} \\
\xi(x) \stackrel{\eta(x)}{\longrightarrow} \xi^{\prime} g(x)
\end{array}
$$

courtesy of the natural transformation $\eta$, from which one can deduce that $(f, \sigma)$ is a morphism of coloured posets. Moreover, it preserves fibres. Thus given a morphism $(g, \eta): \xi \rightarrow \xi^{\prime}$ we have assigned a morphism of coloured posets $\mathcal{E}(g, \eta)=(f, \sigma)$. Since composition behaves well we have

Proposition 1.10. $\mathcal{E}:$ Bund $_{R} \rightarrow \mathcal{C P}_{R}$ is a functor.

There is a homology of coloured posets, and our main interest in this paper is to compute it for the total coloured poset of a given bundle. We now briefly recall the relevant definitions; for more details, see [2]. Let $(P, \mathcal{F})$ be a coloured poset and define a chain complex $\mathcal{S}_{*}(P, \mathcal{F})$ by letting $\mathcal{S}_{k}(P, \mathcal{F})=0$ for $k<0$; for $k>0$ let

$$
\mathcal{S}_{k}(P, \mathcal{F})=\bigoplus_{\substack{x_{1} x_{2} \cdots x_{k} \\ x_{i} \in P \backslash 1}} \mathcal{F}\left(x_{1}\right),
$$

the direct sum over all multisequences $x_{1} \leq x_{2} \leq \cdots \leq x_{k} \in P \backslash 1$, and $\mathcal{S}_{0}(P, \mathcal{F})=$ $\mathcal{F}(1)$. The differential $d_{k}: \mathcal{S}_{k}(P, \mathcal{F}) \rightarrow \mathcal{S}_{k-1}(P, \mathcal{F})$ is defined for $k>1$ by

$$
d_{k}\left(\lambda x_{1} x_{2} \cdots x_{k}\right)=\mathcal{F}\left(x_{1} \leq x_{2}\right)(\lambda) x_{2} \cdots x_{k}-\sum_{i=2}^{k}(-1)^{i} \lambda x_{1} \cdots \widehat{x}_{i} \cdots x_{k},
$$

and $d_{1}$ is defined by $d_{1}(\lambda x)=\mathcal{F}_{x}^{1}(\lambda)$. The homology $H_{*}(P, \mathcal{F})$ of $(P, \mathcal{F})$ is then the homology of this complex, and this gives a functor $H_{*}: \mathcal{C} \mathcal{P}_{R} \rightarrow G r \operatorname{Mod}_{R}$.

When working with homology it can be convenient to use a smaller complex than the one above. One defines $\mathcal{C}_{*}(P, \mathcal{F})$ identically to $\mathcal{S}_{*}(P, \mathcal{F})$, but with the additional requirement that the indexing $x_{1} x_{2} \cdots x_{k}$ appearing above now gives sequences rather than multisequences; i.e., there are no repeats. Clearly $\mathcal{C}_{k} \subset \mathcal{S}_{k}$, and the differential is taken to be the restriction to $\mathcal{C}_{k}$ of the differential on $\mathcal{S}_{k}$ (so that $\mathcal{C}_{*}$ is a subcomplex of $\mathcal{S}_{*}$ ). One can show that there is a homotopy equivalence of chain complexes $\mathcal{C}_{*}(P, \mathcal{F}) \simeq \mathcal{S}_{*}(P, \mathcal{F})$ as in [2, $\left.\S 2\right]$.

Returning to the situation of a bundle $\xi: B \rightarrow \mathcal{C P}_{R}$, these constructions give important colourings of the base. Let $\mathcal{E}(\xi)=(E, \mathcal{F})$. For each positive integer $q \geq 0$ we have the $q$-chain colouring $\mathcal{S}_{q}$ of $B$ : for $x \in B$, let $\mathcal{S}_{q}(x):=\mathcal{S}_{q}\left(E_{x}, \mathcal{F}_{x}\right)$, the module of $q$-chains in the complex $\mathcal{S}_{*}$ of the fibre $\left(E_{x}, \mathcal{F}_{x}\right)$. Recalling from [2, §2] that $\mathcal{S}_{*}$ is a functor $\mathcal{S}_{*}: \mathrm{CP}_{R} \rightarrow \mathrm{Ch}_{R}$, we have that if $x \leq y$ in $B$, then the composition of $\xi: B \rightarrow \mathcal{C} \mathcal{P}_{R}$ with $\mathcal{S}_{q}$ induces a module homomorphism $\mathcal{S}_{q}(x) \rightarrow$ $\mathcal{S}_{q}(y)$, hence gives a coloured poset $\left(B, \mathcal{S}_{q}\right)$.

We can also colour the base using the homology of the fibres. Let $\xi: B \rightarrow \mathcal{C P}_{R}$ with $\mathcal{E}(\xi)=(E, \mathcal{F})$. For any $q$, compose $\xi: B \rightarrow \mathcal{C P}_{R}$ with homology $H_{q}: \mathcal{C P}_{R} \rightarrow$ 
$\mathcal{M}_{R}$ to get a new functor $\mathcal{H}_{q}^{\mathrm{fib}}(\xi)=H_{q} \circ \xi$,

$$
\mathcal{H}_{q}^{\mathrm{fib}}(\xi): B \stackrel{\xi}{\longrightarrow} \mathcal{C} \mathcal{P}_{R} \stackrel{H_{q}}{\longrightarrow} \operatorname{Mod}_{R}
$$

This defines a colouring on $B$ and so a coloured poset $\left(B, \mathcal{H}_{q}^{\mathrm{fib}}(\xi)\right)$. If $(g, \eta): \xi \rightarrow \xi^{\prime}$ is a bundle morphism and $x \in B$, then $\eta(x): \xi(x) \rightarrow \xi^{\prime} g(x)$ induces a map in homology

$$
\eta(x)_{*}: H_{q}(\xi(x)) \rightarrow H_{q}\left(\xi^{\prime} g(x)\right),
$$

which in turn induces a morphism of coloured posets $\left(B, \mathcal{H}_{q}^{\mathrm{fib}}(\xi)\right) \rightarrow\left(B^{\prime}, \mathcal{H}_{q}^{\mathrm{fib}}\left(\xi^{\prime}\right)\right)$. Thus by taking homology of fibres we have a functor $\mathcal{B u n d}_{R} \rightarrow \mathcal{C P} \mathcal{P}_{R}$.

\section{A Bicomplex And its total COMPlex}

Our main theorem is a Leray-Serre type spectral sequence for bundles. Fundamental to its construction is a certain bicomplex which we now describe. Let $\xi: B \rightarrow \mathcal{C P}_{R}$ be a bundle with total coloured poset $\mathcal{E}(\xi)=(E, \mathcal{F})$. From the previous section we have an infinite family of coloured posets $\left(B, \mathcal{S}_{q}\right)$, where the base has been $q$-chain coloured for each $q \geq 0$.

Now set

$$
\mathcal{K}_{p, q}=\mathcal{C}_{p}\left(B, \mathcal{S}_{q}\right) .
$$

Explicitly, $\mathcal{C}_{p}\left(B, \mathcal{S}_{q}\right)$ is a direct sum of modules in the colouring of $B$ indexed by length $p$ sequences in $B \backslash 1$, so a typical element is a sum of $\mu x_{1} \ldots x_{p}$ with the $x_{1}<\cdots<x_{p} \in B \backslash 1$ and $\mu \in \mathcal{S}_{q}\left(E_{x_{1}}, \mathcal{F}_{x_{1}}\right)$. Thus $\mu$ is in turn a sum over $\lambda y_{1} \ldots y_{q}$ with the $y_{1} \leq \cdots \leq y_{q} \in E_{x_{1}} \backslash 1_{x_{1}}$ and $\lambda \in \mathcal{F}_{x_{1}}\left(y_{1}\right)$. We will thus write

$$
\lambda \mathbf{x y}=\lambda x_{1} \ldots x_{p} y_{1} \ldots y_{q}, \quad \lambda \in \mathcal{F}_{x_{1}}\left(y_{1}\right), x_{i} \in B \backslash 1, y_{j} \in E_{x_{1}} \backslash 1_{x_{1}},
$$

for a typical (homogeneous) element of $\mathcal{C}_{p}\left(B, \mathcal{S}_{q}\right)$. Note that $\mathcal{K}_{0, q}=\mathcal{C}_{0}\left(B, \mathcal{S}_{q}\right)=$ $\mathcal{S}_{q}(1)=\bigoplus \mathcal{F}_{1}\left(y_{1}\right)$, the direct sum over the multisequences $y_{1} \ldots y_{q} \in P_{1}$.

For fixed $q$ we have that $\mathcal{K}_{*, q}=\mathcal{C}_{*}\left(B, \mathcal{S}_{q}\right)$ is a complex under the differential $d^{h}: \mathcal{C}_{p}\left(B, \mathcal{S}_{q}\right) \rightarrow \mathcal{C}_{p-1}\left(B, \mathcal{S}_{q}\right)$ given by

$$
\begin{aligned}
d^{h}\left(\lambda x_{1} \ldots x_{p} y_{1} \ldots y_{q}\right)= & \left(\tau_{x_{1}}^{x_{2}}\right)_{y_{1}}(\lambda) x_{2} \ldots x_{p} f_{x_{1}}^{x_{2}}\left(y_{1}\right) \ldots f_{x_{1}}^{x_{2}}\left(y_{q}\right) \\
& -\sum_{i=2}^{p}(-1)^{i} \lambda x_{1} \ldots \widehat{x}_{i} \ldots x_{p} y_{1} \ldots y_{q}
\end{aligned}
$$

where $\left(f_{x_{1}}^{x_{2}}, \tau_{x_{1}}^{x_{2}}\right):\left(E_{x_{1}}, \mathcal{F}_{x_{1}}\right) \rightarrow\left(E_{x_{2}}, \mathcal{F}_{x_{2}}\right)$ is the induced coloured poset morphism.

Fixing $p$ we have a complex under the differential $d^{v}: \mathcal{C}_{p}\left(B, \mathcal{S}_{q}\right) \rightarrow \mathcal{C}_{p}\left(B, \mathcal{S}_{q-1}\right)$,

$$
\begin{aligned}
d^{v}\left(\lambda x_{1} \ldots x_{p} y_{1} \ldots y_{q}\right)=(-1)^{p+q} & \left(\left(\mathcal{F}_{x_{1}}\right)_{y_{1}}^{y_{2}}(\lambda) x_{1} \ldots x_{p} y_{2} \ldots y_{q}\right. \\
& \left.-\sum_{j=2}^{q}(-1)^{j} \lambda x_{1} \ldots x_{p} y_{1} \ldots \widehat{y}_{j} \ldots y_{q}\right) .
\end{aligned}
$$

It is tedious but straightforward to check that $d^{v} d^{h}+d^{h} d^{v}=0$, so that

Lemma 2.1. $\mathcal{K}_{*, *}$ is a bicomplex.

There is then an associated total complex $\left(\mathcal{T}_{*}, d\right)$, where

$$
\mathcal{T}_{n}(E, \mathcal{F}):=\bigoplus_{p+q=n} \mathcal{K}_{p, q}
$$



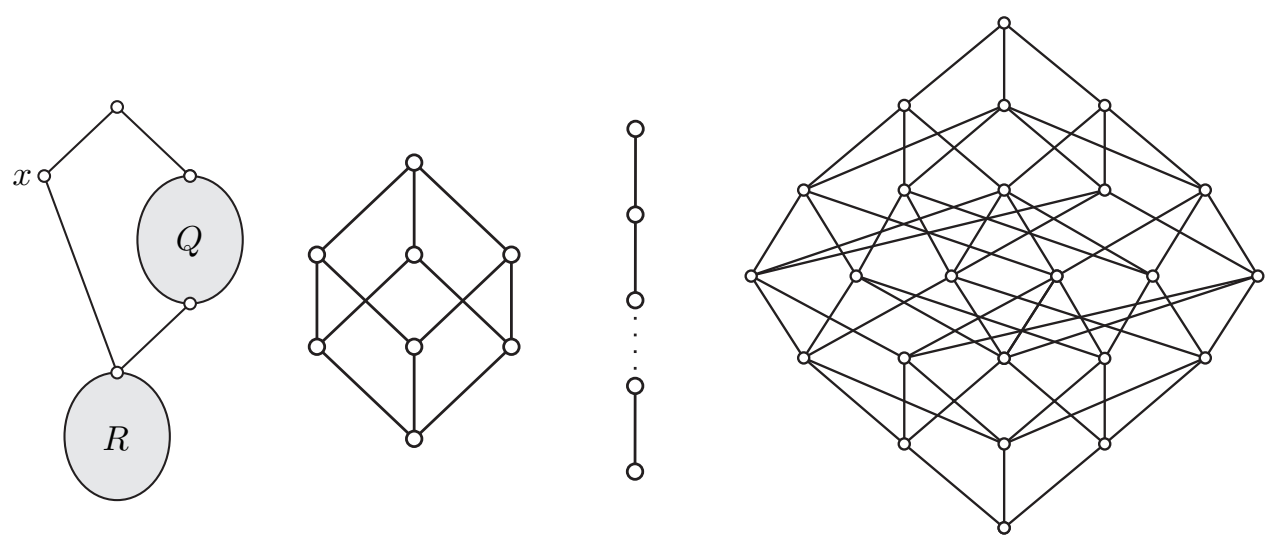

Figure 2. One of these posets is nonadmissible (from left to right): a concocted example where $R$ is any poset with a 1 and $Q$ is any poset with a $0<1$; the Boolean lattice of rank 3 ; the poset $\mathbf{n}=1<2<\cdots<n$; the Bruhat poset of type $A_{3}$.

with $d=d^{h}+d^{v}$. From the general construction of a spectral sequence from a bicomplex we have

Proposition 2.2. Let $\xi$ be a bundle of coloured posets with base $B$ and total poset $(E, \mathcal{F})$. Then there is a spectral sequence

$$
E_{p, q}^{2}=H_{p}\left(B, \mathcal{H}_{q}^{\mathrm{fib}}(\xi)\right) \Longrightarrow H_{*}\left(\mathcal{T}_{*}(E, \mathcal{F})\right) .
$$

Example 2.3. Let $B$ be a poset with a unique minimal element 0 satisfying $0<1$, and let $(P, \mathcal{F})$ be a coloured poset. Then $\mathcal{T}_{*}(B \times(P, \mathcal{F}))$ is acyclic. To see this notice that $\left(B, \mathcal{H}_{q}^{\mathrm{fib}}(\xi)\right)$ is a coloured poset with a constant colouring (by $H_{q}(P, \mathcal{F})$ ) and thus, since $B$ has a 0 , gives [2, Example 8] that the $E_{2}$-page of the above spectral sequence is trivial.

\section{Admissible AND SPECIALLY ADMissible POSETS}

In $₫ 5$ we will identify the $H_{*}\left(\mathcal{T}_{*}(E, \mathcal{F})\right)$ of $₫ 2$ with the $H_{*}(E, \mathcal{F})$ of $₫ 1$ for $(E, \mathcal{F})=$ $\mathcal{E}(\xi)$, where $\xi$ is a bundle over a certain class of posets that we now introduce. Let $P$ be a poset with 1 , and for $x \in P$ recall from $₫ 1$ the definition of the interval $P(x)$ and its complement $\bar{P}(x)$.

Definition 3.1. A poset $P$ with 1 is admissible if and only if there exists an $x \prec 1$ in $P$ such that for all $y \in P(x) \backslash x$, the subposet $L(y):=\{z \in \bar{P}(x) \mid y \leq z\}$ has a unique minimal element $0_{L(y)}$.

On the far left of Figure 2 we have an artificial example of a poset, admissible courtesy of the $x$ shown. In general, $L(y)$ has as its unique maximal element the 1 of $P$ with $0_{L(y)}<1$ by our standing poset convention, so $L(y)$ necessarily contains at least two elements if $P$ is to be admissible. In particular the poset $\mathbf{n}=1<2<\cdots<n$, with Hasse diagram in Figure 2, is nonadmissible when $n \geq 3$.

Example 3.2. For $\mathbb{B}(X)$ the Boolean lattice of all subsets of $X$ ordered by inclusion, $|X|$ is the rank of $\mathbb{B}(X)$. Then $\mathbb{B}(X)$ is admissible for $X \neq \varnothing$. This is vacuous 
in rank 1 (where $\mathbb{B}(X)$ is isomorphic to the poset $\mathbf{2}=1<2$ ). If $|X|>1$, then any subset $A \in \mathbb{B}(X)$ covered by $X$ is of the form $X \backslash x$ for some $x \in X$, and the interval (also a Boolean lattice) $\mathbb{B}(A)$ consists of subsets of $X$ not containing $x$. For $Y \in \mathbb{B}(A) \backslash\{A\}$ the set $L(Y)$ consists of subsets of $X$ containing the element $x$ and the elements of $Y$. Thus $O_{L(Y)}=Y \cup\{x\}$ is a unique minimal element for $L(Y)$. The second example of Figure 2 is Boolean of rank 3.

Example 3.3. Let $(W, S)$ be a Coxeter group with length function $\ell: W \rightarrow \mathbb{Z} \geq 0$ and reflections $T=W^{-1} S W$ (see 3] for basic facts about Coxeter groups). Write $w^{\prime} \rightarrow w$ iff $w=w^{\prime} t$ for some $t \in T$ with $\ell(w)>\ell\left(w^{\prime}\right)$. Define $w^{\prime}<w$ iff there is a sequence $w^{\prime}=w_{0} \rightarrow w_{1} \rightarrow \cdots \rightarrow w_{m}=w$. The result is a graded (by $\ell$ ) poset with 0 (the identity of $W$ ) called the Bruhat (-Chevalley) poset of $W$. A simple example is the Bruhat poset of the Weyl group of type $A_{1}^{n}$, which is the Boolean lattice of rank $n$. Two warnings: $w^{\prime} \rightarrow w$ is not the same as $w^{\prime} \prec w$, and $(W, \leq)$ is not in general a lattice.

If $W$ is finite, then the Bruhat poset also has a 1: the element of longest length in $W$. It turns out that the Bruhat posets for finite $W$ are admissible, though we do not require that generality here. For now, we leave it to the reader to check that the Bruhat poset for the symmetric group $\mathfrak{S}_{4}$ (the Weyl group of type $A_{3}$ ), which is the third example in Figure 2, is admissible for any $x \prec 1$.

Suppose now that $\xi: B \rightarrow \mathcal{C P}_{R}$ is a bundle with admissible base $B$ and $(E, \mathcal{F})=$ $\mathcal{E}(\xi)$. The admissibility of the base has the following useful consequence for $E$. Let $x \in B$, with interval $B(x)$, complement $\bar{B}(x)$, and restricted bundles (see Example 1.8) $\left.\xi\right|_{B(x)},\left.\xi\right|_{B(x)}$ with $E(x)=\mathcal{E}\left(\left.\xi\right|_{B(x)}\right)$ and $\bar{E}(x)=\mathcal{E}\left(\left.\xi\right|_{\bar{B}(x)}\right)$.

Lemma 3.4. Let $B$ be an admissible poset courtesy of $x \prec 1_{B}$. Then for all $y \in E(x) \backslash 1_{E(x)}$, the subposet $J(y)=\{z \in \bar{E}(x) \mid y \leq z\}$ has a unique minimal element.

Proof. We have $\pi(y) \in B(x)$ and by admissibility, the subposet $L(\pi(y))$ has a unique minimal element $z_{0} \in \bar{B}(x)$. The bundle furnishes us with a morphism of coloured posets $\left(E_{\pi(y)}, \mathcal{F}_{\pi(y)}\right) \rightarrow\left(E_{z_{0}}, \mathcal{F}_{z_{0}}\right)$, which is comprised in particular of a poset map $f_{\pi(y)}^{z_{0}}: E_{\pi(y)} \rightarrow E_{z_{0}}$. We leave it to the reader to verify that the minimal element we seek is $f_{\pi(y)}^{z_{0}}(y) \in E_{z_{0}}$.

Admissible posets are an important intermediary concept: the bundle results of $\$ 4$ are for instance true when the base is admissible. For the main theorem of \$5 we require something stronger: that the base poset $B$ is admissible for some $x \prec 1$, and when split into the associated interval $B(x)$ and its complement $\bar{B}(x)$, these two are also admissible, and when these are split the results are admissible, and so on. Thus, we will employ an inductive approach, resulting in a collection of posets scattered on the workbench, and we will need each piece to be admissible. The definition is best formulated recursively:

Definition 3.5. A poset $P$ with 1 is specially admissible if and only if either

- $P$ is Boolean of rank 1 , or

- $P$ is admissible for some $x \prec 1$ with $P(x)$ and $\bar{P}(x)$ specially admissible.

Example 3.6. We have already seen that the Boolean lattice of rank $n>0$ is admissible (for any $x \prec 1$ ) with the $P(x)$ and $\bar{P}(x)$ Boolean of rank $n-1$. The 


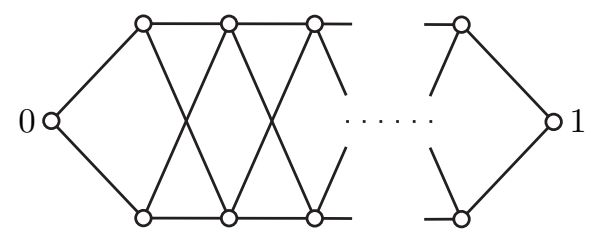

Figure 3. Hasse diagram for the Bruhat poset of type $I_{2}(m)$, laid out on its side with the ordering running from left to right.

Boolean lattice of rank 1 is specially admissible by definition, so Boolean lattices of any rank are specially admissible by induction.

Example 3.7. Another simple family of specially admissible posets are the Bruhat posets of type $I_{2}(m), m \geq 2$, which have Hasse diagrams (laid out sideways with order running from left to right) as in Figure 3. For any $x \prec 1$, the interval $P(x)$ is the Bruhat poset of type $I_{2}(m-1)$, and its complement $\bar{P}(x)$ is Boolean of rank 1. As any two elements in $I_{2}(m)$ are comparable, we have that for $y \in P(x) \backslash x$, the poset $L(y)$ is also Boolean of rank 1. Thus $I_{2}(m)$ is admissible. The Bruhat poset of type $I_{2}(2)$ is the Boolean lattice of rank 2, and so by induction, $I_{2}(m)$ is specially admissible.

Example 3.8. Looking back at Figure 2, one can see (after quite an inspection) that the Bruhat poset $A_{3}$ is specially admissible. Indeed, it turns out that the Bruhat posets for finite $W$ are specially admissible.

\section{Some EXACT SEQUEnCES}

This section contains the technical tools needed for the proof of the main theorem. As we will see in $\$ 5$ the key step is the identification of the homology of $\mathcal{T}_{*}(E, \mathcal{F})$ with the coloured poset homology $H_{*}(E, \mathcal{F})$ in the case where the base poset is specially admissible. To achieve this we will use an inductive argument, splitting the base into two pieces and then using the two long exact sequences presented below in Propositions 4.1 and 4.2 .

Throughout this section let $B$ be a poset, admissible courtesy of some $x \prec 1_{B}$. Let $\xi$ be a bundle of coloured posets with base $B$ and $\mathcal{E}(\xi)=(E, \mathcal{F})$. Let $B(x)$ be the interval from $\$ 1$ and $\bar{B}(x)$ its complement, with the associated restricted bundles having total posets $(E(x), \mathcal{F})$ and $(\bar{E}(x), \mathcal{F})$.

Proposition 4.1. Let $\xi: B \rightarrow \mathcal{C P}_{R}$ be a bundle of coloured posets with $B$ admissible, $(E, \mathcal{F})$ the total coloured poset and $\mathcal{T}_{*}(E, \mathcal{F})$ the total complex of $\oiint_{2}$. Then there is a long exact sequence,

$$
\cdots \stackrel{\delta}{\longrightarrow} H_{n}\left(\mathcal{T}_{*}(\bar{E}(x), \mathcal{F})\right) \longrightarrow H_{n}\left(\mathcal{T}_{*}(E, \mathcal{F})\right) \longrightarrow H_{n-1}\left(\mathcal{T}_{*}(E(x), \mathcal{F})\right) \stackrel{\delta}{\longrightarrow} \cdots .
$$

Proof. It is immediate from the definition of the differential that $\mathcal{T}_{*}(\bar{E}(x), \mathcal{F})$ is a subcomplex of $\mathcal{T}_{*}(E, \mathcal{F})$ and thus for some quotient $Q_{*}^{\prime}$ there is a short exact sequence

$$
0 \longrightarrow \mathcal{T}_{*}(\bar{E}(x), \mathcal{F}) \longrightarrow \mathcal{T}_{*}(E, \mathcal{F}) \longrightarrow Q_{*}^{\prime} \longrightarrow 0
$$


Explicitly, $Q_{*}^{\prime}$ can be described as

$$
Q_{n}^{\prime}=\bigoplus_{\mathbf{x z y}} \mathcal{F}_{x_{1}}\left(y_{1}\right)
$$

the sum over $\mathbf{x z y}=x_{1} \ldots x_{i} z_{1} \ldots z_{j} y_{1} \ldots y_{k}$ with $i>0$ and $i+j+k=n$, and where $x_{1} \ldots x_{i}$ is a sequence in $B(x), z_{1} \ldots z_{j}$ a sequence in $\bar{B}(x) \backslash 1$, and $y_{1} \ldots y_{k}$ a multisequence in $E_{x_{1}} \backslash 1_{E_{x_{1}}}$. Here, as elsewhere, $E_{x_{1}}$ is the fibre over $x_{1}$. Notice that the condition $i>0$ means that the sequence $x_{1} \ldots x_{i} z_{1} \ldots z_{j}$ must start in $B(x)$. When $i>1$ the differential in $Q_{*}^{\prime}$ sends $\lambda$ xyz to

$$
\begin{aligned}
& \left(\tau_{x_{1}}^{x_{2}}\right)_{y_{1}}(\lambda) x_{2} \ldots x_{i} z_{1} \ldots z_{j} f_{x_{1}}^{x_{2}}\left(y_{1}\right) \ldots f_{x_{1}}^{x_{2}}\left(y_{k}\right) \\
& -\sum_{s=2}^{i}(-1)^{s} \lambda x_{1} \ldots \widehat{x}_{s} \ldots x_{i} z_{1} \ldots z_{j} y_{1} \ldots y_{k} \\
& \quad-\sum_{t=1}^{j}(-1)^{i+t} \lambda x_{1} \ldots x_{i} z_{1} \ldots \widehat{z}_{t} \ldots z_{j} y_{1} \ldots y_{k} \\
& \quad+(-1)^{n}\left(\mathcal{F}_{x_{1}}\right)_{y_{1}}^{y_{2}}(\lambda) x_{1} \ldots x_{i} z_{1} \ldots z_{j} y_{2} \ldots y_{k} \\
& \quad-\sum_{\ell=2}^{k}(-1)^{\ell+n} \lambda x_{1} \ldots x_{i} z_{1} \ldots z_{j} y_{1} \ldots \widehat{y}_{\ell} \ldots y_{k}
\end{aligned}
$$

and for $\lambda x z_{1} \ldots z_{j} y_{1} \ldots y_{k}$ the image is

$$
\begin{aligned}
\sum_{t=1}^{j}(-1)^{t} \lambda & x z_{1} \ldots \widehat{z}_{t} \ldots z_{j} y_{1} \ldots y_{k} \\
+ & (-1)^{n}\left(\mathcal{F}_{x}\right)_{y_{1}}^{y_{2}}(\lambda) x z_{1} \ldots z_{j} y_{2} \ldots y_{k} \\
& -\sum_{\ell=2}^{k}(-1)^{\ell+n} \lambda x z_{1} \ldots z_{j} y_{1} \ldots \widehat{y}_{\ell} \ldots y_{k} .
\end{aligned}
$$

Now let $D_{*}^{\prime}$ be the submodule of $Q_{*}^{\prime}$ consisting of the modules indexed by the $\mathbf{x z y}=x_{1} \ldots x_{i} z_{1} \ldots z_{j} y_{1} \ldots y_{k}$ with $x_{i} \neq x$. In other words, the last element of the sequence in $B(x)$ is not the maximum element $x$ of this interval. It is immediate that $D_{*}^{\prime}$ is a subcomplex of $Q_{*}^{\prime}$ and thus for the quotient $A_{*}^{\prime}$ there is a short exact sequence

$$
0 \longrightarrow D_{*}^{\prime} \longrightarrow Q_{*}^{\prime} \stackrel{q^{\prime}}{\longrightarrow} A_{*}^{\prime} \longrightarrow 0 .
$$

We show that $D_{*}^{\prime}$ is acyclic, and this allows us to finish the proof easily: one sees that

$$
\pi^{\prime}: A_{i+k}^{\prime} \rightarrow \mathcal{T}_{i+k-1}(E(x), \mathcal{F})
$$

given by $\lambda x_{1} \ldots x_{i-1} x y_{1} \ldots y_{k} \mapsto \lambda x_{1} \ldots x_{i-1} y_{1} \ldots y_{k}$ is an isomorphism, and so using the long exact sequence associated to (4.2), we have $H_{n}\left(Q_{*}^{\prime}\right) \cong H_{n-1}\left(\mathcal{T}_{*}(E(x)\right.$, $\mathcal{F})$ ). Plugging this into the long exact sequence associated to (4.1) gives the result.

The remainder of the proof is thus devoted to showing that $D_{*}^{\prime}$ is acyclic. We filter $D_{*}^{\prime}$ as follows:

$$
F_{s} D_{n}^{\prime}=\bigoplus_{\mathbf{x z y}} \mathcal{F}_{x_{1}}\left(y_{1}\right)
$$


where the sum is over the $\mathbf{x z y}=x_{1} \ldots x_{i} z_{1} \ldots z_{j} y_{1} \ldots y_{k}$ satisfying $1 \leq i \leq s$. There is an associated spectral sequence converging to $H_{*}\left(D_{*}^{\prime}\right)$ having

$$
E_{s, t}^{0}=\bigoplus_{\mathbf{x z y}} \mathcal{F}_{x_{1}}\left(y_{1}\right)
$$

where the sum is over the $\mathbf{x z y}=x_{1} \ldots x_{s} z_{1} \ldots z_{j} y_{1} \ldots y_{k}$ with $j+k=t$. The differential $d^{0}$ sends $\lambda x_{1} \ldots x_{s} z_{1} \ldots z_{j} y_{1} \ldots y_{k}$ to

$$
\begin{aligned}
-\sum_{i=1}^{j}(-1)^{s+i} \lambda x_{1} \ldots x_{s} z_{1} \ldots \widehat{z}_{i} \ldots z_{j} y_{1} \ldots y_{k} & \\
+ & (-1)^{n}\left(\mathcal{F}_{x_{1}}\right)_{y_{1}}^{y_{2}}(\lambda) x_{1} \ldots x_{s} z_{1} \ldots z_{j} y_{2} \ldots y_{k} \\
& \quad-\sum_{i=2}^{k}(-1)^{n+i} \lambda x_{1} \ldots x_{s} z_{1} \ldots z_{j} y_{1} \ldots \widehat{y}_{i} \ldots y_{k} .
\end{aligned}
$$

Given $\mathbf{x}=x_{1} \ldots x_{s}$ a sequence in $B(x) \backslash x$ we define $U_{*}^{\mathbf{x}} \subseteq E_{s, *}^{0}$ to be the submodule of elements of the form $\lambda x_{1} \ldots x_{s} z_{1} \ldots z_{j} y_{1} \ldots y_{k}$, where the sequence $x_{1} \ldots x_{s}$ is the given one $\mathbf{x}$. Endowing this with the differential gives a subcomplex.

Let $L\left(x_{s}\right)$ be the subposet of $B$ with elements $\left\{z \in \bar{B}(x) \mid x_{s} \leq z\right\}$ as in Definition 3.1 and having a unique minimal element courtesy of the admissibility of $B$. By direct comparison of the definitions on both sides we see that there is an isomorphism of complexes

$$
U_{*}^{\mathrm{x}} \cong \mathcal{T}_{*-s}\left(L\left(x_{s}\right) \times E_{x_{1}}\right),
$$

and by Example 2.3, the right-hand side (and hence $U_{*}^{\mathbf{x}}$ ), is acyclic.

We now show that any cycle in $E_{s, *}^{0}$ is a boundary. A typical cycle has the form $\sigma=\sum \lambda_{\mathbf{x z y}} \mathbf{x z y}$ and can be decomposed as

$$
\sigma=\sum \sigma^{\mathbf{x}}
$$

where $\sigma^{\mathbf{x}}=\sum_{\mathbf{x}^{\prime}=\mathbf{x}} \lambda_{\mathbf{x}^{\prime} \mathbf{z y}} \mathbf{x}^{\prime} \mathbf{z y}$. Now, if $\mathbf{x z y}$ and $\mathbf{x}^{\prime} \mathbf{z}^{\prime} \mathbf{y}^{\prime}$ are two sequences occurring in $\sigma$ and $\mathbf{x} \neq \mathbf{x}^{\prime}$, then $d^{0}(\mathbf{x z y})$ and $d^{0}\left(\mathbf{x}^{\prime} \mathbf{z}^{\prime} \mathbf{y}^{\prime}\right)$ are in disjoint summands of $d(\sigma)$. Thus $d^{0}(\sigma)=0$ implies that $d^{0}\left(\sigma^{\mathbf{x}}\right)=0$ for all $\sigma^{\mathbf{x}}$. In other words, as $\sigma$ is a cycle, each $\sigma^{\mathbf{x}}$ is a cycle. Clearly we have $\sigma^{\mathbf{x}} \in U_{*}^{\mathbf{x}}$ and hence since $U_{*}^{\mathbf{x}}$ is acyclic, there exists $\tau^{\mathbf{x}}$ such that $d^{0}\left(\tau^{\mathbf{x}}\right)=\sigma^{\mathbf{x}}$. Thus $d^{0}\left(\sum \tau^{\mathbf{x}}\right)=\sum \sigma^{\mathbf{x}}=\sigma$ and $\sigma$ is a boundary as claimed.

Thus, for each $s$, the complex $E_{s, *}^{0}$ is acyclic and hence the $E^{1}$-page of the spectral sequence is trivial, showing that $D_{*}^{\prime}$ is acyclic as required.

In the proof of the main theorem in $\$ 5$, we will find it more convenient to use

$$
(-1)^{k} \pi^{\prime}: \lambda x_{1} \ldots x_{i-1} x y_{1} \ldots y_{k} \mapsto(-1)^{k} \lambda x_{1} \ldots x_{i-1} y_{1} \ldots y_{k}
$$

as an isomorphism $A_{*}^{\prime} \rightarrow \mathcal{T}_{*}(E(x), \mathcal{F})$. We leave it to the reader to check that this is indeed an isomorphism.

There is a similar long exact sequence for the homology of the total coloured poset $(E, \mathcal{F})$. It is a generalization of [2, Theorem 1] from bases that are Boolean of rank 1 to bundles over admissible bases. The proof is very similar to the last proposition so we will be briefer. 
Proposition 4.2. Let $\xi: B \rightarrow \mathcal{C P}_{R}$ be a bundle of coloured posets with $B$ admissible and total coloured poset $(E, \mathcal{F})$. Then there is a long exact sequence

$$
\cdots \stackrel{\delta}{\longrightarrow} H_{n}(\bar{E}(x), \mathcal{F}) \longrightarrow H_{n}(E, \mathcal{F}) \longrightarrow H_{n-1}(E(x), \mathcal{F}) \stackrel{\delta}{\longrightarrow} \cdots .
$$

Proof. There is a short exact sequence

$$
0 \longrightarrow \mathcal{S}_{*}(\bar{E}(x), \mathcal{F}) \longrightarrow \mathcal{S}_{*}(E, \mathcal{F}) \longrightarrow Q_{*} \longrightarrow 0,
$$

and as above we can describe $Q_{*}$ explicitly as

$$
Q_{n}=\bigoplus_{\mathbf{x z}} \mathcal{F}\left(x_{1}\right),
$$

where $\mathbf{x z}=x_{1} \ldots x_{i} z_{1} \ldots z_{j}$ with $i>0, i+j=n$ and $x_{1} \ldots x_{i}$ a multisequence in $E(x)$ and $z_{1} \ldots z_{j}$ a multisequence in $\bar{E}(x) \backslash 1_{E}$.

Now let $D_{*}$ be the subcomplex of $Q_{*}$ consisting of the modules indexed by the $\mathbf{x z}=x_{1} \ldots x_{i} z_{1} \ldots z_{j}$ such that $x_{i} \neq 1_{E(x)}$, and let $A_{*}$ be the quotient of $Q_{*}$ by $D_{*}$ with quotient map $q$. We show, as above, that $D_{*}$ is acyclic, so that if

$$
\pi: A_{i+j} \rightarrow \mathcal{S}_{i+j-1}(E(x), \mathcal{F})
$$

is the isomorphism $\lambda x_{1} \ldots x_{i-1} 1_{E(x)} z_{1} \ldots z_{j} \mapsto \lambda x_{1} \ldots x_{i-1} z_{1} \ldots z_{j}$, then $\pi q$ is a quasi-isomorphism from $Q_{*}$ to $\mathcal{S}_{*-1}(E(x), \mathcal{F})$.

Filter $D_{*}$ by

$$
F_{s} D_{n}=\bigoplus_{\mathbf{x z}} \mathcal{F}\left(x_{1}\right)
$$

where the sum is over the $\mathbf{x z}=x_{1} \ldots x_{i} z_{1} \ldots z_{j}$ satisfying $1 \leq i \leq s$. The associated spectral sequence has

$$
E_{s, t}^{0}=\bigoplus_{\mathbf{x z}} \mathcal{F}\left(x_{1}\right),
$$

where the sum is over $\mathbf{x z}=x_{1} \ldots x_{s} z_{1} \ldots z_{t}$. The differential $d^{0}$ is as follows (where the overall sign is given by the parity of $s$ ):

$$
\pm d^{0}\left(\lambda x_{1} \ldots x_{s} z_{1} \ldots z_{t}\right)=\sum_{k=1}^{t}(-1)^{i} \lambda x_{1} \ldots x_{s} z_{1} \ldots \widehat{z}_{i} \ldots z_{t} .
$$

Fixing $\mathbf{x}=x_{1} \ldots x_{s}$ a multisequence in $E_{x} \backslash 1_{E(x)}$, we define $V_{*}^{\mathbf{x}} \subseteq E_{s, *}^{0}$ to be the subcomplex of elements of the form $\lambda x_{1} \ldots x_{s} z_{1} \ldots z_{j}$, where the multisequence $x_{1} \ldots x_{s}$ is the given one $\mathbf{x}$. Let $J\left(x_{s}\right)$ be the subposet of $E$ with elements $\{z \in$ $\left.\bar{E}_{x} \mid x_{s} \leq z\right\}$. By Lemma 3.4, $J\left(x_{s}\right)$ has a 0 and $V_{*}^{\mathbf{x}} \cong \mathcal{S}_{*-s}\left(J\left(x_{s}\right), \mathcal{F}\left(x_{1}\right)\right)$. Since the poset $J\left(x_{s}\right)$ is coloured constantly by $\mathcal{F}\left(x_{1}\right)$, we conclude by [2, Example 8 and Corollary 1] that $V^{\mathbf{x}}$ is acyclic. The remainder of the proof is more or less identical to the previous proposition.

\section{A Leray-Serre spectral Sequence}

Here is the main theorem of the paper.

Theorem 5.1. Let $\xi: B \rightarrow \mathrm{CP}_{R}$ be a bundle of coloured posets with $B$ finite and specially admissible and $(E, \mathcal{F})$ the associated total coloured poset. Then there is a spectral sequence

$$
E_{p, q}^{2}=H_{p}\left(B, \mathcal{H}_{q}^{\mathrm{fib}}(\xi)\right) \Longrightarrow H_{*}(E, \mathcal{F}) .
$$




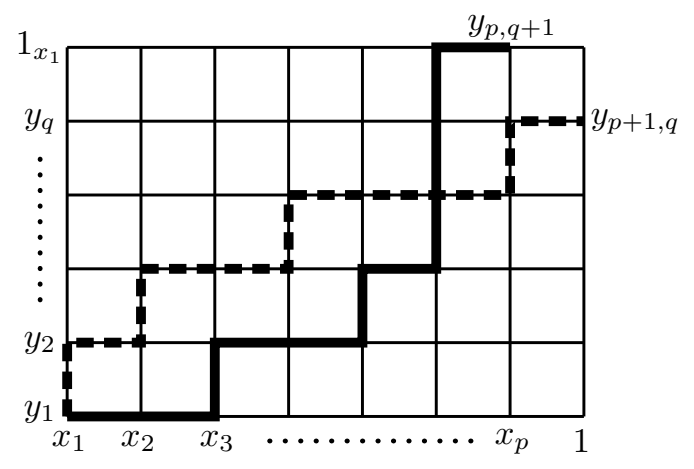

FiguRE 4. (x, y)-grid with two possible multisequences $\mathbf{z}$.

The strategy of the proof is as follows: in $₫ 2$ we constructed a spectral sequence, which by Proposition 2.2 converges,

$$
E_{p, q}^{2}=H_{p}\left(B, \mathcal{H}_{q}^{\mathrm{fib}}(\xi)\right) \Longrightarrow H_{*}\left(\mathcal{T}_{*}(E, \mathcal{F})\right) .
$$

It suffices then to find a quasi-isomorphism $\phi: \mathcal{T}_{*}(E, \mathcal{F}) \rightarrow \mathcal{S}_{*}(E, \mathcal{F})$, inducing an isomorphism $H_{*}\left(\mathcal{T}_{*}(E, \mathcal{F})\right) \cong H_{*}\left(\mathcal{S}_{*}(E, \mathcal{F})\right)=H_{*}(E, \mathcal{F})$.

We begin by defining a chain map $\phi: \mathcal{T}_{*}(E, \mathcal{F}) \rightarrow \mathcal{S}_{*}(E, \mathcal{F})$. Let $\xi: B \rightarrow \mathcal{C P}_{R}$ be a bundle, with $B$ any base, and let $(E, \mathcal{F})$ be the total coloured poset. Let $(\mathbf{x}, \mathbf{y})$ be a pair consisting of a sequence $\mathbf{x}=x_{1}<\cdots<x_{n}$ in $B$ and a multisequence $\mathbf{y}=y_{1} \leq \cdots \leq y_{m}$ in $E_{x_{1}}$. Note that we allow $1 \in B$ to be an element of $\mathbf{x}$ and $1_{x_{1}} \in E_{x_{1}}$ to be an element of $\mathbf{y}$. For each $1 \leq i \leq n$, the bundle furnishes us with a poset map $f_{x_{1}}^{x_{i}}: E_{x_{1}} \rightarrow E_{x_{i}}$, so let $y_{i j}=f_{x_{1}}^{x_{i}}\left(y_{j}\right)$, giving

$$
y_{i 1} \leq \cdots \leq y_{i m}
$$

a multisequence in $E_{x_{i}}\left(\right.$ with $\left.y_{1 j}=y_{j}\right)$. The resulting $m \times n$ collection $\left\{y_{i j}\right\}$ may be placed on the vertices of a rectangular array, and we join these by placing oriented edges between $y_{i, j}$ and $y_{i, j+1}$ and between $y_{i, j}$ to $y_{i+1, j}$. We refer to this as the grid associated to the pair $(\mathbf{x}, \mathbf{y})$. We have comparability of elements in the columns of the grid, $y_{i j} \leq y_{i, j+1}$, thanks to (5.1), and also along the rows, $y_{i j}<y_{i+1, j}$, via the definition of the ordering on $E$.

Given $x_{1}<\cdots<x_{p} \in B \backslash 1$ and $y_{1} \leq \cdots \leq y_{q} \in E_{x_{1}} \backslash 1_{x_{1}}$ with $p, q>0$, we consider the grid associated to $\left(\mathbf{x}=x_{1}<\cdots<x_{p}<1, \mathbf{y}=y_{1} \leq \cdots \leq y_{q}<1_{x_{1}}\right)$. An (x, y)-multisequence is a multisequence in $E$ of the form,

$$
\mathbf{z}=z_{1} \leq \cdots \leq z_{p+q}
$$

with the $z_{k}$ elements of the grid satisfying $z_{1}=y_{11}, z_{p+q}=y_{p+1, q}$ or $z_{p+q}=y_{p, q+1}$, and for any $1 \leq k \leq p+q$, if $z_{k}=y_{i j}$, then $z_{k+1} \in\left\{y_{i+1, j}, y_{i, j+1}\right\}$. In other words an $(\mathbf{x}, \mathbf{y})$-multisequence consists of the ordered collection of vertices in an oriented path from $y_{11}$ to either $y_{p+1, q}$ or $y_{p, q+1}$. Figure 4 exhibits a grid and two sample paths.

Such a path partitions the grid into two halves: the upper-left half and the lower-right half. Given an $(\mathbf{x}, \mathbf{y})$-multisequence $\mathbf{z}$ we define $m(\mathbf{z})$ to be the number of squares of the grid in the lower-right half. 
We now define the map $\phi$ on the generator $\lambda \mathbf{x y}=\lambda x_{1} \cdots x_{p} y_{1} \cdots y_{q}$ by

$$
\phi(\lambda \mathbf{x y})=(-1)^{\alpha(q)} \sum_{\mathbf{z}}(-1)^{m(\mathbf{z})} \lambda \mathbf{z},
$$

where the sum is over all $(\mathbf{x}, \mathbf{y})$-multisequences $\mathbf{z}$, and $\alpha(q)=1$ when $q \equiv 1,2 \bmod 4$, or $\alpha(q)=0$ otherwise (alternatively, $(-1)^{\alpha(q)}=(-1)^{[q]}$ for $[q]=1+2+\cdots+q$ ).

Note that (5.2) is also defined when $p$ or $q$ is 0 : we have $\mathcal{T}_{p+0}(E, \mathcal{F})=\mathcal{C}_{p}\left(B, \mathcal{S}_{0}\right)$ with a typical element $\lambda x_{1} \ldots x_{p}$, where $\lambda \in \mathcal{F}_{x_{1}}\left(1_{x_{1}}\right)$ and $\phi(\lambda \mathbf{x})=\lambda 1_{x_{1}} \ldots 1_{x_{p}}$. Similarly $\mathcal{T}_{0+q}=\mathcal{C}_{0}\left(B, \mathcal{S}_{q}\right)$ with typical element $\lambda y_{1} \ldots y_{q}$ for $\lambda \in \mathcal{F}_{1}\left(y_{1}\right)$, and $\phi(\lambda \mathbf{y})=(-1)^{\alpha(q)} \lambda y_{1} \ldots y_{q}$. Finally, note that $\mathcal{T}_{0+0}=\mathcal{F}_{1}\left(1_{P_{1}}\right)=\mathcal{S}_{0+0}$, so by convention we define $\phi=$ id : $\mathcal{T}_{0+0} \rightarrow \mathcal{S}_{0+0}$.

Proposition 5.2. $\phi$ is a chain map with $\phi\left(\mathcal{T}_{*}(\bar{E}(x), \mathcal{F})\right) \subset \mathcal{S}_{*}(\bar{E}(x), \mathcal{F})$ for $x \in B$.

Proof. The inclusion of complexes is clear, so it remains to show that $\phi d_{\mathcal{T}}(\lambda \mathbf{x y})$ $=d_{\delta} \phi(\lambda \mathbf{x y})$ for any $\lambda \mathbf{x y} \in \mathcal{T}_{p+q}(E, \mathcal{F})$. There are two parts to the proof: first we show that every term of $\phi d_{\mathcal{T}}$ also occurs as a term of $d_{\S} \phi$, and then that any extra terms in $d_{s} \phi$ occur in \pm pairs, and hence cancel.

Ignoring signs for the moment, the terms of $\phi d_{\mathcal{T}}$ are precisely the terms of $d_{\S} \phi$ that are obtained by taking a $\mathbf{z}=z_{1} \ldots z_{p+q}$ of $\phi(\lambda \mathbf{x y})$ and letting the differential $d_{\S}$ omit $z_{i}$ as
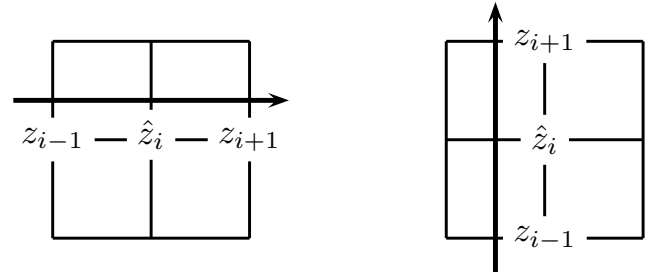

or by letting $d_{\S}$ omit the first element $z_{1}$ or the last element $z_{p+q}$. In words, if $d_{\S}$ omits $z_{i}$, then $z_{i-1}, z_{i+1}$ are both in the same row, or both in the same column, as $z_{i}$. Indeed, the situation above left arises in $\phi d_{\mathcal{T}}$ when $d_{\mathcal{T}}$ omits some $x \in B$ that indexes the column in which $z_{i}$ lies, and then mapping to the multisequence $\hat{\mathbf{z}}=z_{1} \ldots \widehat{z}_{i} \ldots z_{p+q}$ corresponding to a path in the grid in which the $i$-th column of the original grid has been deleted. The situation above right is similar, with an appropriate $y \in E_{x_{1}}$ omitted by $d_{\mathcal{T}}$ this time. Chasing definitions one can verify that the coefficients of such terms agree up to sign.

With that out of the way, the terms of $d_{\mathcal{S}} \phi$ that don't arise at all in $\phi d_{\mathcal{T}}$ are those where $d_{\mathcal{S}}$ omits from a multisequence $\mathbf{z}$ a $z_{i}$ sitting on a "corner":

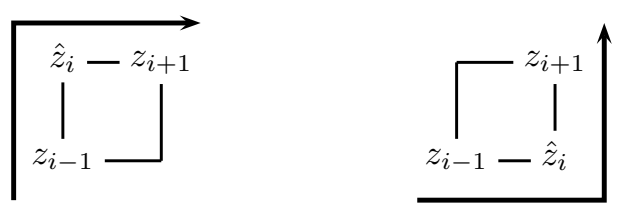

Now to the signage, where we check first that these extra terms occur in \pm pairs and so cancel. Suppose we have such a term, arising when $d_{\S}$ omits a corner from a $\mathbf{z} \in \phi(\lambda \mathbf{x y})$. There is a unique repetition of this term, arising by taking the $\mathbf{z}^{\prime} \in \phi(\lambda \mathbf{x y})$ that travels the other way around this square and letting $d_{\S}$ omit the other corner ( $\mathbf{z}$ and $\mathbf{z}^{\prime}$ thus differ only in the segment shown in the figure above). It is easy to check that $\mathbf{z}, \mathbf{z}^{\prime}$ acquire different signs from $\phi$ and that $d_{\mathfrak{S}}$ preserves this difference. Thus, the extra terms in $d_{s} \phi$ can be coupled into \pm pairs as required. 


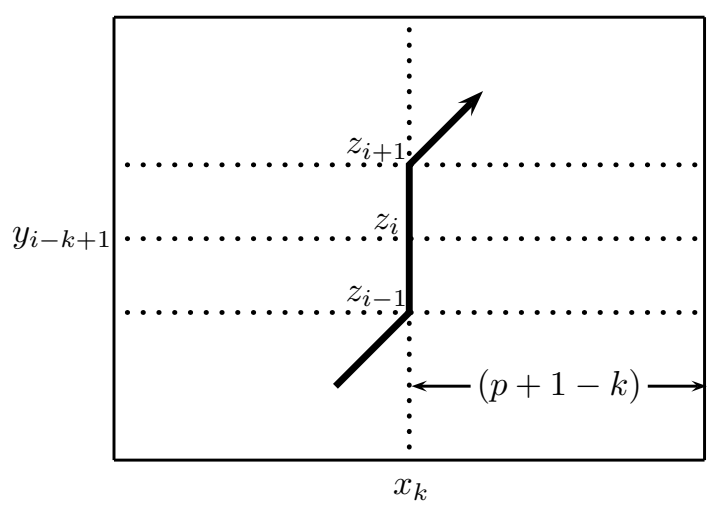

FiguRE 5. Schematic $\mathbf{z}$ with $z_{i-1}, z_{i}, z_{i+1}$ all in the same column.

Now to the terms common to $\phi d_{\mathcal{T}}$ and $d_{\mathcal{S}} \phi$, where we show that they occur with the same signs. Let $\lambda \mathbf{x y}=\lambda x_{1} \cdots x_{p} y_{1} \cdots y_{q}$ and apply $\phi$ to give a sum of $(\mathbf{x}, \mathbf{y})$-multisequences with the multisequence $\mathbf{z}$ having $\operatorname{sign}(-1)^{\alpha(q)+m(\mathbf{z})}$. Now applying $d_{\mathcal{S}}$ to this we get a sum of terms of the form $z_{1} \ldots \hat{z}_{i} \ldots z_{p+q}$ picking up a sign $-(-1)^{i}$. Thus the total sign of the summand of $d_{\mathcal{S}} \phi(\lambda \mathbf{x y})$ indexed by $z_{1} \ldots \hat{z}_{i} \ldots z_{p+q}$ is

$$
-(-1)^{\alpha(q)+m(\mathbf{z})+i} \text {. }
$$

Now there are two cases to consider, where the first is if $z_{i-1}, z_{i}$ and $z_{i+1}$ are all in the same column (see Figure 5).

Suppose the column in question is that of $x_{k}$ for some $k$. This implies that the row corresponding to $z_{i}$ is that indexed by $y_{i-k+1}$. Thus in order to obtain via $\phi d_{\mathcal{T}}$ the summand indexed by $z_{1} \ldots \hat{z}_{i} \ldots z_{p+q}$ we must as a first step consider the summand of $d_{\mathcal{T}}(\lambda \mathbf{x y})$ indexed by the term $x_{1} \cdots x_{p} y_{1} \cdots \hat{y}_{i-k+1} \cdots y_{q}$. This picks up the sign $-(-1)^{p+q+(i-k+1)}$. After applying $\phi$ the summand indexed by $\hat{\mathbf{z}}=z_{1} \ldots \hat{z}_{i} \ldots z_{p+q}$ picks up an additional $(-1)^{\alpha(q-1)+m(\hat{\mathbf{z}})}$ so the total sign is

$$
-(-1)^{p+q+(i-k+1)+\alpha(q-1)+m(\hat{\mathbf{z}})} .
$$

Now, $\hat{\mathbf{z}}$ lives in a new grid obtained from the original one by deleting the $(i-k+1)$-th row. Thus

from which we deduce

$$
m(\hat{\mathbf{z}})=m(\mathbf{z})-(p+1-k)
$$

$$
p+q+(i-k+1)+\alpha(q-1)+m(\hat{\mathbf{z}})=\alpha(q-1)+m(\mathbf{z})+i .
$$

By checking the various possibilities for $\alpha(q)$ (or by observing that $q+[q-1]=[q]$ ), we see that the signs of (5.3) and (5.4) coincide. The second case, where $z_{i-1}, z_{i}$ and $z_{i+1}$ are all in the same row, is entirely similar.

As a matter of interest we note that $\phi$ is the unique chain map of this form. Thinking degree by degree, if $\phi$ is to be a chain map, then its definition at $\mathcal{T}_{p+q}$ is determined by its definition at $\mathcal{T}_{p-1, q}$ and $\mathcal{T}_{p, q-1}$, as well as the differentials $d_{\mathcal{T}}$ and $d_{\mathcal{S}}$. Thus, once we have defined $\phi$ at $\mathcal{T}_{0+0}$, we have no choice for the definition at $\mathcal{T}_{0+1}, \mathcal{T}_{1+0}$, and these in turn determine the definition at $\mathcal{T}_{1+1}$, and so on, working our way upwards and rightwards from the bottom left-hand corner of the bicomplex. 
As $\mathcal{T}$ and $\mathcal{S}$ coincide in degree 0 , the simplest choice for $\phi$ is the identity, and our map is precisely the chain map determined by this choice.

Proposition 5.3. $\phi$ is a quasi-isomorphism when $B$ is finite and specially admissible.

Proof. Let $B$ be admissible via $x \prec 1$. Recalling the notation used in $₫ 4$ there is a morphism of short exact sequences:

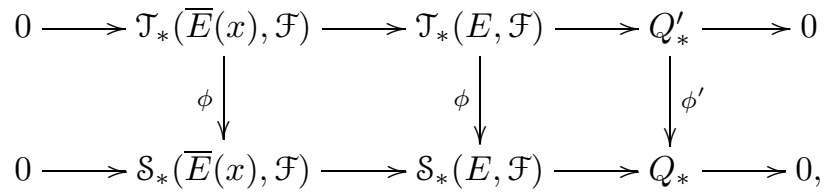

where $\phi^{\prime}$ is the map induced on the quotients by the second part of Proposition 5.2. It is easy to check that the diagram commutes. We thus have a commutative diagram containing the following portion:

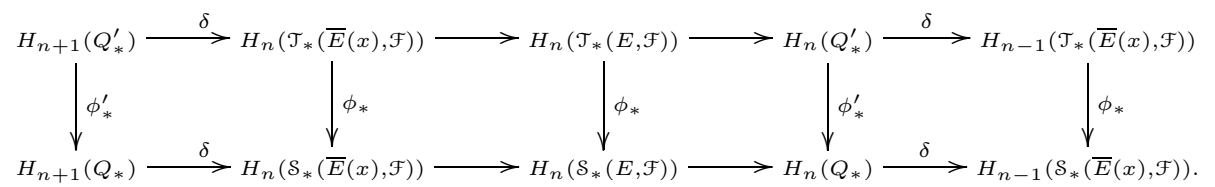

We leave it to the reader to check that the diagram

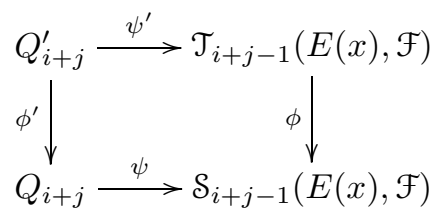

commutes, with $\psi:=\pi q$ and $\psi^{\prime}:=(-1)^{j} \pi^{\prime} q^{\prime}$ the quasi-isomorphisms of $\$ 4$ In particular, we have $\phi_{*}^{\prime}=\psi_{*}^{-1} \phi_{*} \psi_{*}^{\prime}$.

We now argue by induction on the cardinality of the base. If $B$ is Boolean of rank one with two elements $0<1$, then $B$ is admissible for $x=0, E(x)$ is the fibre $\left(E_{0}, \mathcal{F}_{0}\right)$ and $\bar{E}(x)$ is the fibre $\left(E_{1}, \mathcal{F}_{1}\right)$. Both of these are the total spaces of a bundle over the trivial poset with a single element. For such a bundle, $\mathcal{T}_{*}=\mathcal{S}_{*}$ and $\phi=\mathrm{id}$, inducing an isomorphism $H_{*} \mathcal{T}_{*} \rightarrow H_{*} \mathcal{S}_{*}$. Thus, we have the result for $B$ using the diagrams above and the 5-lemma.

In general, as $B$ is specially admissible, so are $B(x)$ and $\bar{B}(x)$, and so the proof is again finished by induction and the 5-lemma.

The proof of Theorem [5.1] is now also complete: Proposition 2.2 gives us a spectral sequence

$$
E_{p, q}^{2}=H_{p}\left(B, \mathcal{H}_{q}^{F}(\xi)\right) \Longrightarrow H_{*}\left(\mathcal{T}_{*}(E, \mathcal{F})\right),
$$

and Proposition 5.3 gives us an isomorphism $H_{*}\left(\mathcal{T}_{*}(E, \mathcal{F})\right) \cong H_{*}(E, \mathcal{F})$.

We remark here that our proof relies heavily on having a specially admissible base because in general the long exact sequences used in the previous proposition do not exist. Whether the theorem remains true in greater generality remains an open question. 


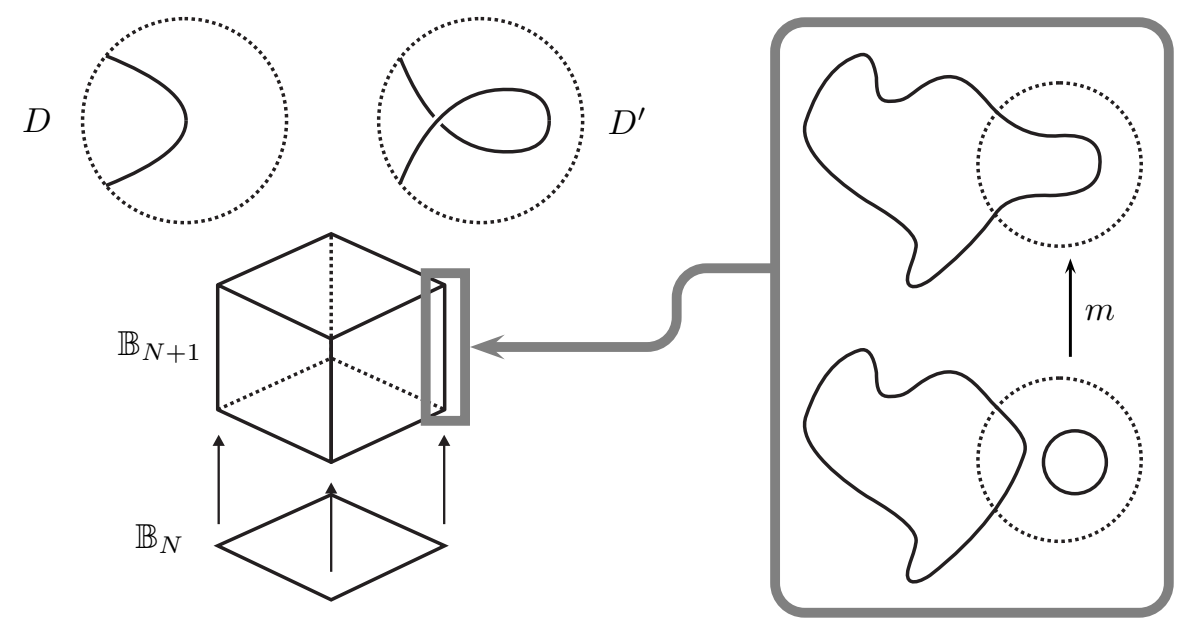

Figure 6. Decomposing a Khovanov-coloured Boolean lattice $\mathbb{B}_{N+1}$ as a bundle over $\mathbb{B}_{N}$ to show invariance of the first Reidemeister move.

Example 5.4. The bundle perspective and the spectral sequence above provide an alternative proof of invariance under the first Reidemeister move for Khovanov homology. Let $D$ and $D^{\prime}$ be link diagrams, identical except in a small disc as on the top left of Figure 6 .

For a link diagram $D$ with $N$ crossings, let $\mathcal{F}_{D}$ be the Khovanov colouring of the Boolean lattice $\mathbb{B}_{N}$ on the crossings. To show invariance under Reidemeister I, we require $H_{*+1}\left(\mathbb{B}_{N+1}, \mathcal{F}_{D^{\prime}}\right) \cong H_{*}\left(\mathbb{B}_{N}, \mathcal{F}_{D}\right)$.

Consider the crossings of $D^{\prime}$ outside the disk. We can decompose $\left(\mathbb{B}_{N+1}, \mathcal{F}_{D^{\prime}}\right)$ as a bundle over the Boolean lattice $\mathbb{B}_{N}$ on these crossings: the fibres correspond to the two possible resolutions of the crossing in the disk as in Figure 6 (with $n=2$ ).

In particular, the complex $\mathcal{C}_{*}$ for the fibres is $\mathrm{id}^{\otimes i} \otimes m \otimes \mathrm{id}^{\otimes j}: V^{\otimes i+j+2} \rightarrow$ $V^{\otimes i+j+1}$, where $V$ is the Frobenius algebra used in the definition of Khovanov homology and $m$ is its multiplication. As $m$ is onto and $\operatorname{ker}(m) \cong V$ we have that $\mathcal{H}_{*}^{\mathrm{fib}}(\xi)$ is $V$ in degree 1 and 0 elsewhere; thus the homology colouring $\left(\mathbb{B}_{N}, \mathcal{H}_{1}^{\mathrm{fib}}(\xi)\right)$ of the base is $\left(\mathbb{B}_{N}, \mathcal{F}_{D}\right)$. The spectral sequence thus has $E^{2}$-page

$$
E_{p, q}^{2}= \begin{cases}H_{p}\left(\mathbb{B}_{N}, \mathcal{F}_{D}\right), & q=1, \\ 0, & \text { otherwise }\end{cases}
$$

collapsing, and so $H_{*-1}\left(\mathbb{B}_{N}, \mathcal{F}_{D}\right) \cong H_{*}\left(\mathbb{B}_{N+1}, \mathcal{F}_{D^{\prime}}\right)$. Now apply the main theorem of [2], turning this isomorphism into the required one between the Khovanov homologies.

\section{An application to KNOT homology}

In this section we give an application of the spectral sequence of Theorem 5.1 to knot homology. We will write the results in terms of Khovanov homology, but in fact one can be more general; see the remarks at the end of the section.

The idea is the following. Starting with a link diagram we choose a subset of the crossings to be fixed and form a cube of link diagrams by resolving the remaining 

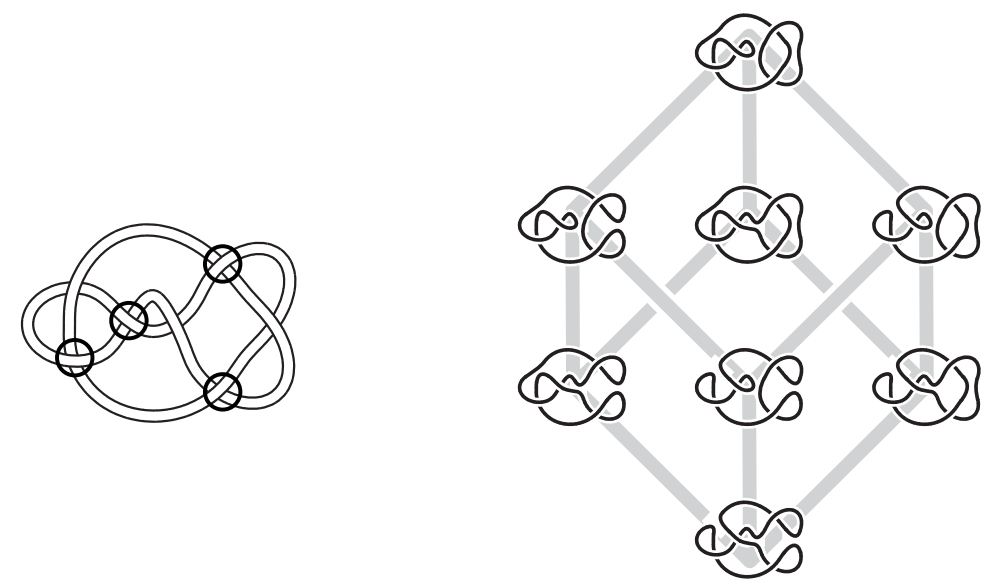

Figure 7 . The knot $7_{5}$ with four fixed crossings.

crossings in the way familiar in Khovanov homology (see Khovanov 4 and BarNatan [1]). We are now at liberty to take the Khovanov homology of each link diagram at a cube vertex and moreover to consider the induced maps associated to Morse moves along the edges. From this we can form a complex à la Khovanov. The main result is that there is a spectral sequence converging to the Khovanov homology of the original diagram with the homology of this new complex at the $E^{2}$-page.

Our conventions for shifting complexes will be that for a tri-graded vector space $W_{*, *, *}$ we define $W_{*, * *}[a, b, c]$ by $\left(W_{*, *, *}[a, b, c]\right)_{i, j, k}=W_{i-a, j-b, k-c}$. Similar conventions apply to bi-graded and singly graded vector spaces.

First we outline our grading and normalisation conventions in constructing Khovanov homology. Let $D$ be a link diagram with $N$ crossings. The unnormalised Khovanov homology of the diagram $D$, denoted $\overline{K H}_{*, *}(D)$, is defined to be the homology of the complex $\overline{\mathcal{C}}_{*, *}(D)$ given as

$$
\overline{\mathfrak{C}}_{i, *}(D)=\bigoplus_{\mathrm{rk}(\alpha)=N-i} V^{\otimes k_{\alpha}}[\operatorname{rk}(\alpha)]
$$

where $V$ is the usual graded vector space defining Khovanov homology, $k_{\alpha}$ is the number of circles appearing in smoothing $\alpha$ and $\mathrm{rk}$ is the rank function on the Boolean lattice. The differential $d: \overline{\mathrm{e}}_{i, *}(d) \rightarrow \overline{\mathrm{C}}_{i-1, *}$ is the usual one.

If the diagram $D$ is oriented, with $N_{+}$positive and $N_{-}$negative crossings, we can also define the normalised Khovanov homology to be

$$
K H_{*, *}(D)=\overline{K H}_{*, *}(D)\left[-N_{+}, N_{+}-2 N_{-}\right]
$$

The reader should note that this differs from the original conventions followed by Khovanov 4 and Bar-Natan 1, where unnormalised homology is defined via a cochain complex $\overline{\mathcal{C}}^{*, *}(D)$ which is normalised to define $K H^{*, *}(D)$, the latter being an invariant of oriented links. The conventions we use are related to the original 
ones by

$$
\begin{gathered}
\overline{\mathcal{C}}^{i, j}(D)=\overline{\mathcal{C}}_{N-i, j}(D), \\
K H^{i, j}(D)=K H_{-i, j}(D) .
\end{gathered}
$$

Now we consider the complex referred to above. Let $D$ be an oriented link diagram with $N$ crossings. Choose $k$ crossings $c_{1}, \ldots, c_{k}$ and call them the fixed crossings. The remaining $\ell=N-k$ crossings are the free crossings, and we assume these too have been ordered.

Resolving the free crossings in one of the two familiar ways yields $2^{\ell}$ diagrams which we place at the vertices of the Boolean lattice $\mathbb{B}_{\ell}$ (a cube in more usual Khovanovology). Figure 7 illustrates this for the knot $7_{5}$ with the four fixed crossings as shown in red.

For $x \in \mathbb{B}_{\ell}$ let $D_{x}$ denote the corresponding link diagram. Note that $D_{x}$ does not in general inherit an orientation from $D$, and we will be treating $D_{x}$ as an unoriented diagram.

We now associate to each vertex $x$ of the cube the bi-graded module

$$
V_{*, *}(x)=\overline{K H}_{*, *}\left(D_{x}\right)[0, \operatorname{rk}(x)],
$$

the unnormalised Khovanov homology of the (unoriented) diagram $D_{x}$ shifted in the second degree by the rank of $x$ in $\mathbb{B}_{\ell}$.

If $x<x^{\prime} \in \mathcal{B}_{\ell}$, then the diagrams $D_{x}$ and $D_{x^{\prime}}$ are identical except in a small disc within which one of the zero smoothings in $D_{x}$ turns into a 1-smoothing in $D_{x^{\prime}}$. By using the multiplication or comultiplication in the familiar way (geometrically using the saddle cobordism) there is a chain map,

$$
\overline{\mathcal{C}}_{*, *}\left(D_{x}\right) \rightarrow \overline{\mathcal{C}}_{*, *}\left(D_{x^{\prime}}\right) .
$$

This map has bi-degree $(0,-1)$. Thus, after taking homology and shifting, we have a homomorphism of bi-degree $(0,0)$,

$$
\delta_{x}^{x^{\prime}}: V_{*, *}(x) \rightarrow V_{*, *}\left(x^{\prime}\right) .
$$

We now define the complex $\overline{\mathcal{K}}_{*, *, *}\left(D ; c_{1}, \ldots, c_{k}\right)$ by setting

$$
\overline{\mathcal{K}}_{i, *, *}\left(D ; c_{1}, \ldots, c_{k}\right)=\bigoplus_{x \in \mathbb{B}_{\ell}, \mathrm{rk}(x)=N-i} V_{*, *}(x) .
$$

The differential is defined using the maps $\delta_{x}^{x^{\prime}}$ in the usual way (and using the usual signage conventions in Khovanov homology). It has tri-degree $(1,0,0)$, and is indeed a differential because all squares in the cube anti-commute (even though these are now Morse-move frames in a movie of link diagrams) because saddles can be rearranged without any unwanted side effects.

We now define the Khovanov homology of the diagram $D$ with respect to the given fixed crossings to be

$$
\overline{K H}_{*, *, *}\left(D ; c_{1}, \ldots, c_{k}\right):=H\left(\overline{\mathcal{K}}_{*, *, *}\left(D ; c_{1}, \ldots, c_{k}\right)\right) .
$$

It is important to note that $\overline{K H}_{*, *, *}\left(D ; c_{1}, \ldots, c_{k}\right)$ depends on the set of fixed crossings and is with respect to a specific diagram. However, $\overline{K H}_{*, *, *}\left(D ; c_{1}, \ldots, c_{k}\right)$ is invariant under reordering of the fixed and free crossings. If the set of crossings is empty then we recover the unnormalised Khovanov homology.

With these preliminaries let us now state the theorem. 
Theorem 6.1. Let $D$ be an $N$-crossing link diagram and let $c_{1}, \ldots, c_{k}$ be $k$ crossings of $D$. For each $i$, there is a spectral sequence

$$
E_{p, q}^{2}=\overline{K H}_{p, q, i}\left(D ; c_{1}, \ldots, c_{k}\right) \Longrightarrow \overline{K H}_{p+q, i}(D) .
$$

The $r$-th differential in the spectral sequence has bi-degree $(-r, r-1)$.

Proof. The procedure for constructing $\overline{K H}_{*, *}(D)$ includes (in our language) the Khovanov colouring $\mathcal{F}$ of the Boolean lattice $\mathbb{B}_{k+\ell}$, on the set of crossings of the diagram. It is a graded colouring in that it takes values in graded modules.

We have been given a subset of the crossings in the form of the fixed crossings. By Example 1.7 we can decompose $\left(\mathbb{B}_{k+\ell}, \mathcal{F}\right)$ as a bundle $\xi$ over the Boolean lattice on the free crossings, i.e. a copy of $\mathbb{B}_{\ell}$. Note that the total space of this bundle is $\left(\mathbb{B}_{k+\ell}, \mathcal{F}\right)$.

Fixing $i$ and applying Theorem 5.1 we thus have a spectral sequence

$$
E_{p, q}^{2}=H_{p}\left(\mathbb{B}_{\ell}, \mathcal{H}_{q}^{\mathrm{fib}}(\xi)\right) \Longrightarrow H_{*, i}\left(\mathbb{B}_{k+\ell}, \mathcal{F}\right)
$$

where the colouring $\mathcal{F}$ is the restriction of the Khovanov colouring to grading $i$.

Recall from 2 that

$$
\overline{K H}_{*, *}(D) \cong H_{*, *}\left(\mathbb{B}_{k+\ell}, \mathcal{F}\right) .
$$

Thus the spectral sequence above converges as required to $\overline{K H}_{*, i}(D)$, and it remains to identify the $E^{2}$-page.

It is clear that for $x \in \mathbb{B}_{\ell}$ the fibre over $x$ is isomorphic to $\mathbb{B}_{k}$ equipped with the Khovanov colouring $\mathcal{F}_{x}$ of the diagram $D_{x}$ shifted by $\operatorname{rk}(x)$. Thus the homology of the fibre over $x$ is given by $H_{*, *}\left(\mathbb{B}_{k}, \mathcal{F}_{x}\right)[0, \mathrm{rk}(x)]$. Using again the isomorphism between Khovanov homology and coloured poset homology, we see that the homology of the fibre is isomorphic to $\overline{K H}_{*, *}\left(D_{x}\right)[0, \operatorname{rk}(x)]=V_{*, *}(x)$. The induced maps between fibres are by definition the maps $\delta_{x}^{x^{\prime}}$. Thus we have coloured $\mathbb{B}_{\ell}$ by the modules $V_{*, *}(x)$ and the maps $\delta_{x}^{x^{\prime}}$. The $E^{2}$-page of the spectral sequence is the coloured poset homology of this coloured poset and $\overline{K H}_{*, *, i}\left(D ; c_{1}, \ldots, c_{k}\right)$ is the Khovanov homology of it. Appealing again to 2, these two homologies agree, and hence the result is proved.

Remark 6.2. In practice it may well be useful to apply Reidemeister moves to $D_{x}$, and a quick look at Figure 7 should be enough to see why. Since we are using unnormalised Khovanov homology to colour fibres we must be a little careful as shifts need to be introduced. The best way to proceed is to choose an orientation for $D_{x}$ and count the number of positive and negative crossings $n_{+}$and $n_{-}$. One can then express $V_{*, *}(x)$ in terms of normalised Khovanov homology as

$$
V_{*, *}(x)=K H_{*, *}\left(D_{x}\right)\left[n_{+}, 2 n_{-}-n_{+}+\operatorname{rk}(x)\right],
$$

and now of course we are free to compute $K H_{*, *}\left(D_{x}\right)$ by applying Reidemeister moves if necessary.

Remark 6.3. All of the above works in more generality than is stated. Indeed one can do a similar thing given a link homology theory which can be extended to knotted resolutions. An important example of this is Khovanov-Rozansky theory, which is constructed from a cube of resolutions where each resolution can be viewed as a 4-valent graph. By fixing some subset of crossings we get a (smaller) cube of knotted 4-valent graphs. Khovanov-Rozansky homology has been extended to such knotted graphs by Wagner in [5]. By following through the procedure above one 
then gets a spectral sequence converging to Khovanov-Rozansky homology with a diagram-dependent, but in principle calculable, $E^{2}$-page.

\section{REFERENCES}

[1] Dror Bar-Natan, On Khovanov's categorification of the Jones polynomial, Algebr. Geom. Topol. 2 (2002), 337-370 (electronic). MR1917056 (2003h:57014)

[2] Brent Everitt and Paul Turner, Homology of coloured posets: a generalisation of Khovanov's cube construction, J. Algebra 322 (2009), no. 2, 429-448. MR2529096 (2010h:06005)

[3] James E. Humphreys, Reflection groups and Coxeter groups, Cambridge Studies in Advanced Mathematics, vol. 29, Cambridge University Press, Cambridge, 1990. MR.1066460 (92h:20002)

[4] Mikhail Khovanov, A categorification of the Jones polynomial, Duke Math. J. 101 (2000), no. 3, 359-426. MR1740682 (2002j:57025)

[5] Emmanuel Wagner, Sur l'homologie de Khovanov-Rozansky des graphes et des entrelacs, Ph.D. Thesis (2008).

Department of Mathematics, University of York, York YO10 5DD, England

E-mail address: brent.everitt@york.ac.uk

Département de mathématiques, Université de Fribourg, CH-1700 Fribourg, Switzerland - And - Section de mathématiques, Université de Genève, 2-4 Rue du Lièvre, CH1211, Geneva, Switzerland

E-mail address: prt.maths@gmail.com 